\title{
Ring polymer simulations with global radius of curvature
}

\author{
T. Neuhaus, ${ }^{1, *}$ O. Zimmermann, ${ }^{1, \dagger}$ and Ulrich H. E. Hansmann ${ }^{1,2, \ddagger}$ \\ ${ }^{1}$ John von Neumann Institute for Computing, FZ Jülich, D-52425 Jülich, Germany \\ ${ }^{2}$ Department of Physics, Michigan Technological University, 1400 Townsend Drive, Houghton, Michigan 49931-1295, USA
}

(Received 13 July 2006; revised manuscript received 23 November 2006; published 23 May 2007)

\begin{abstract}
We simulate three-dimensional flexible off-lattice ring polymers of length $L$ up to $L=4000$ for various values of the global radius of curvature $R_{g r c}=0.25,0.48$, and 1.0 and $R_{g r c}=2.0$. We utilize two different ensembles: one with a $\delta$-function constraint on the radius, and the other with a $\theta$-function. For both cases the global radius of curvature provides a valid regularization of polymers with thickness $D=2 R_{g r c}$. The Flory-type critical exponent $\nu_{S A W}$ of self-avoiding rings at $D=2$ is found to be $\nu_{S A W}=0.5869(5)$ from the radii of gyration chain length scaling, while other $D$ values produce consistent results. For our current implementation, the numerical effort of chain thickness calculations is bounded by a number $\mathcal{O}(L \ln L)$ per single update. We also study low-temperature configurations of spatially dense Lennard-Jones homopolymers on a ring and identify some conformational building blocks.
\end{abstract}

DOI: 10.1103/PhysRevE.75.051803

PACS number(s): 36.20.Fz, 82.35.Pq, 36.20.Ey, 05.40.Fb

\section{INTRODUCTION}

Despite decades of research the mechanism by which proteins fold into their biologically active form has still not been resolved [1]. Most of the ongoing work focuses on the sequence specificity of the final structure, i.e., emphasizes the differences between proteins and polymers. For instance, in the funnel picture [2] it is assumed that the energy landscape of a functional protein resembles a funnel leading to the biologically active structure, while a random chain leads to the rough energy landscape of spin glasses. Recently, a different approach has been considered. Banavar et al. [3-5] claimed that ground state conformations of short and thick homopolymers exhibit only a finite set of conformational building blocks, i.e., helices, sheets, and some others, which are independent of the actual form of the chain interactions employed. This suggests a picture where protein folding uses an underlying tendency of thick polymers to form helices, sheets, and other local elements. While this picture does not contradict the funnel hypothesis, it emphasizes the basic polymer (and therefore sequence-independent) characteristics of protein folding. The role of sequence specificity is then to modulate this basic mechanism and to determine the position and ordering of the secondary structure elements.

In light of the claims by Banavar et al. it seems to be useful to study the physics of thick polymers in more detail. Such investigations could lead to insights into the folding mechanism of proteins but may also help to overcome a major drawback in present protein simulations: the lack of sufficiently accurate energy functions [6,7]. A natural starting point is homopolymers, i.e., three-dimensional (3D) offlattice chains with a constant finite thickness $D$, tubes. We will consider nonattractive flexible tubes-without explicit stiffness term—and in a more ambitious approach thick ho-

\footnotetext{
*Corresponding author.

Electronic address: t.neuhaus@fz-juelich.de

${ }^{\dagger}$ Electronic address: o.zimmermann@fz-juelich.de

"Electronic address: hansmann@mtu.edu
}

mopolymers with an attractive interaction on the chain. In this paper, we discuss some basic theoretical and Monte Carlo simulation aspects of tubes, which are regularized via the global radius of curvature, which in 1999 was introduced by Gonzalez and Maddocks [8] as a particular elegant approach to tube thickness. Alternative and slightly different definitions of tube thickness have been discovered and rediscovered during that time, see $[9,10]$, with the earliest contribution likely to be from Krötenheerdt and Veit in 1976 [11].

In one part of this work we study ring polymers at finite thickness with emphasis on the field theoretic long chain limit. This limit of the thick ring polymer, on physical grounds, is expected to be in the universality class of selfavoiding rings, which fairly certain is identical [12-14] to the universality class of self-avoiding walks, i.e., the universality class of physical polymers. The current restriction to rings is intended to prepare future studies on the count of knots in thick polymeric or biologic matter, which by itself is an interesting principal area of research, see, e.g., [15-17].

Simulating rings of length $L$ up to $L=4000$ with good statistics we study volume exclusion for long ring polymers with thicknesses as regularized through global radius of curvature. For the self-avoidance exponent we find the value

$$
\nu_{S A W}=0.5869(5),
$$

which is close to and consistent with the recently published values $\nu_{S A W}=0.5877(6)$ [18], $\nu_{S A W}=0.5874(2)$ [19], and $\nu_{S A W}=0.58765(20)[20]$ and the unpublished value $\nu_{S A W}$ $=0.58758(7)$ from Nickel [21]. We will also present an exploratory study of the tubed Lennard-Jones homopolymer in an attempt to identify some ground state conformational building blocks of the finite length polymer as a function of the thickness.

The paper is organized as follows: Section II introduces the global radius of curvature for chains, the models, and simulation techniques, while Sec. III presents our numerical results. Section IV concludes the paper. 


\section{TUBES, THICKNESS, MODELS, AND SIMULATION}

\section{A. Thickness and the global radius of curvature}

The chain thickness of a polymer is a purely geometrical concept and as a first starting point it should suffice to attach some volume valued geometrical objects, like spheres-pearl necklace - or cylinders to the backbone of the chain. Their surfaces would approximate the tube. Such an approach, however, soon turns out to be impractical for long chains, and presumably is also plagued by nonuniversalities of the actual construction principle for the tubes surface. Mathematicians have discussed tubes in the context of knot theory $[8,10,11]$. Given an open-ended rope with thickness $D$, one determines for a given knot class $K$ the minimal rope length $\tilde{L}(K)$, which allows for the ropes closure, i.e., the formation of a ring. A proper and correct thickness definition then has the property that the thickness of the trivial knot is related via

$$
D=\frac{1}{\pi} \tilde{L}(K=\text { trivial })
$$

to its minimal length. Such a definition using the global radius of curvature can in fact be given [8].

For any three noncollinear points $\mathbf{x}_{a}, \mathbf{x}_{b}$, and $\mathbf{x}_{c}$ in $\mathcal{R}^{3}$, there exists a unique circle through these points with radius of curvature $R_{r c}$ equal to [8]

$$
R_{r c}\left(\mathbf{x}_{a}, \mathbf{x}_{b}, \mathbf{x}_{c}\right)=\frac{d_{a b} d_{a c} d_{b c}}{4 A_{\text {triangle }}\left(\mathbf{x}_{a}, \mathbf{x}_{b}, \mathbf{x}_{c}\right)}
$$

Here, $d_{a b}=\left|\mathbf{x}_{a}-\mathbf{x}_{b}\right|$ and similarly $d_{a c}$ and $d_{b c}$ denote Euclidean distances and $A_{\text {triangle }}\left(\mathbf{x}_{a}, \mathbf{x}_{b}, \mathbf{x}_{c}\right)$ is the area of the triangle spanned by $\mathbf{x}_{a}, \mathbf{x}_{b}$, and $\mathbf{x}_{c}$

$$
A_{\text {triangle }}\left(\mathbf{x}_{a}, \mathbf{x}_{b}, \mathbf{x}_{c}\right)=\sqrt{s\left(s-d_{a b}\right)\left(s-d_{a c}\right)\left(s-d_{b c}\right)}
$$

with

$$
s=\frac{d_{a b}+d_{a c}+d_{b c}}{2},
$$

or alternatively:

$$
A_{\text {triangle }}\left(\mathbf{x}_{a}, \mathbf{x}_{b}, \mathbf{x}_{c}\right)=\frac{1}{2}\left|\left(\mathbf{x}_{a}-\mathbf{x}_{b}\right) \times\left(\mathbf{x}_{a}-\mathbf{x}_{c}\right)\right|
$$

with $\times$ denoting the outer vector product. We will consider $3 \mathrm{D}$ chains with length $L=(N-1) a$ and $a=1$ with configurations

$$
\operatorname{conf}=\left[\mathbf{x}_{0}, \ldots, \mathbf{x}_{N}\right]
$$

and the constraint

$$
\mathbf{x}_{0}=\mathbf{x}_{N}
$$

putting the polymer on a ring. (In general we consider rings to be special cases of polymers with open ends. We therefore use the open ended polymers dependence of the length $L$ on the number $N$ of monomers.) In particular, a ring of length 10 is made out of 11 different monomers. In the following, we identify the 3-tuples $\mathbf{x}_{a}, \mathbf{x}_{b}$, and $\mathbf{x}_{c}$ of Eq. (2.2) with the monomer positions on the chain, and thus in effect we con- sider a discretized version of polymer tubes, which in later refinements could be replaced by a continuum model (using virtual monomers on the chain bonds for a finer tube regularization). The radius of curvature for three consecutive nodes $\mathbf{x}_{i}, \mathbf{x}_{j=1+\bmod (i, N)}$, and $\mathbf{x}_{k=1+\bmod (i+1, N)}$ on the ring with $i$ $=1, \ldots, N(0$ is identified with $N)$ is denoted the local radius of curvature $R_{l r c}$ and is related to the bending angle $\theta_{b}$

$$
\theta_{b}=\cos ^{-1}\left[\left(\mathbf{x}_{i+2}-\mathbf{x}_{i+1}\right)\left(\mathbf{x}_{i+1}-\mathbf{x}_{i}\right)\right]
$$

via

$$
\theta_{b}=\frac{1}{R_{l r c}}+O\left(R_{l r c}^{-3}\right)
$$

for small angles. It is instructive to study the solutions to the "3-body" inequality

$$
R_{r c}(\mathbf{x}, \mathbf{y}, \mathbf{z})>\hat{R}_{0},
$$

which, without loss of generality, can be obtained via translations, rotations, and scale transformations from the solutions to

$$
R_{r c}\left(\mathbf{x}=-\frac{1}{2} \mathbf{e}_{1}, \mathbf{y}=\frac{1}{2} \mathbf{e}_{1}, \mathbf{z}\right)>R_{0} .
$$

As twice the radius of curvature is bounded from below by the distance $d_{12}=1$ between $\mathbf{x}$ and $\mathbf{y}, 1 \leqslant 2 R_{r c}$, any arbitrary $\mathbf{z} \in \mathcal{R}^{3}$ is a solution for values $R_{0} \leqslant 1 / 2$. For values $R_{0}$ $>1 / 2$ there exists an excluded volume region, whose surface is generated by rotations of $R_{0}$ circles through $\mathbf{x}$ and $\mathbf{y}$ around the $\mathbf{x}-\mathbf{y}$ direction. A two-dimensional plot of the solution set $\mathbf{z}$ to the problem Eq. (2.11) at $R_{0}=1.4>1 / 2$ is depicted in Fig. 1 for purposes of illustration.

The global radius of curvature is the minimum of all local radii

$$
R_{g r c}:=\min \left\{R_{r c}\left(\mathbf{x}_{i}, \mathbf{x}_{j}, \mathbf{x}_{k}\right)\right\}
$$

with $1 \leqslant i<j<k \leqslant N$ on that set. It is a scalar quantity, which is nonlocal in nature and in principal requires a number of $O\left(L^{3}\right)$ triangle area calculations. Given that construction, solutions to the constraint

$$
R_{g r c}>R_{0}
$$

also solve the constraints

$$
R_{r c}\left(\mathbf{x}_{\mathbf{i}}, \mathbf{x}_{\mathbf{j}}, \mathbf{x}_{\mathbf{k}}\right)>R_{0}
$$

for all $1 \leqslant i<j<k \leqslant N$ individually, while given the equality

$$
R_{g r c}=R_{0}
$$

at least one incidence in addition satisfies

$$
R_{r c}\left(\mathbf{x}_{\mathbf{i}}, \mathbf{x}_{\mathbf{j}}, \mathbf{x}_{\mathbf{k}}\right)=R_{0} .
$$

For both types of constraints, any chain segment-at node numbers $i$ and $i+1$ - creates a volume exclusion with respect to any other node on the chain, similar to that of Fig. 1. A two-dimensional plot of one solution manifold $\mathbf{z}$ to Eq. (2.13), one node at $\mathbf{z}$ and an $L=9$ open-ended chain in a plane, is depicted in Fig. 2. The chain thickness is defined as $D=2 R_{0}$. It is locally determined by the chains stiffness as 


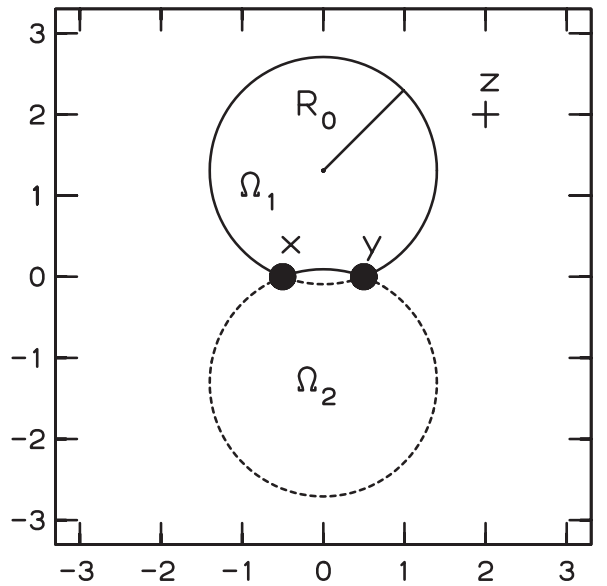

FIG. 1. The solution set $\mathbf{z}$ of the inequality Eq. (2.11) for $R_{0}$ $=1.4$. The positions of $\mathbf{x}$ and $\mathbf{y}$ are marked by solid circles and are associated with two nodes on a chain. Any third point $\mathbf{z}$, which neither falls into $\Omega_{1}$ nor $\Omega_{2}$ solves Eq. (2.11). A possible solution is indicated by the single cross in the figure. The areas $\Omega_{1}$ and $\Omega_{2}$ are bounded through solid and dashed circle sections, respectively, as drawn in the figure. Consequently there is a small lens-shaped area allowed along the chains backbone, provided $R_{0}$ is not too small. In $3 \mathrm{D}$ the excluded volume is generated upon rotating the figure around the 1-axis. The surface of the 3D excluded volume has two parts, an outer surface generated by the longer circle segments and an inner surface generated by the shorter ones. For discretized tubes at $a=1$ and $R_{0}<0.5$ the excluded volume vanishes.

given in Eq. (2.9), while at the same time the approach of distant tube sections is contained in Eq. (2.12).

The current thickness definition via the three-point function of Eq. (2.2) suffers from the apparent defficiency, that for thickness values of $D \leqslant 1$ the simple tube picture breaks down. In this "unphysical" region self-avoidance no longer is enforced in the approach of some monomer towards a tube, but rather it is required that three nonadjacent monomers can not come very close to one another, which on the classical level does not have any physical analog. We like to emphasize that statistical theories built around three-point functions in any case must be viewed as effective theories. Their sole purpose is the correct modeling of nature for some limited parameter range, which, however, we attempt to identify within the scope of the present work.

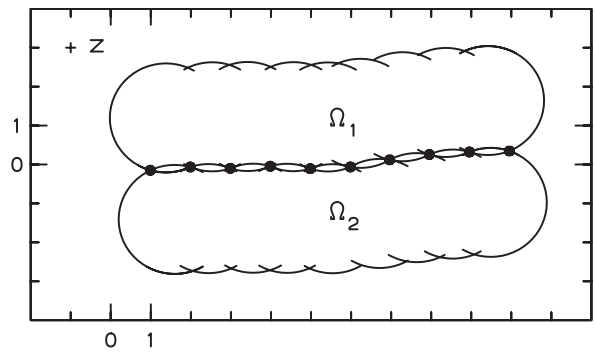

FIG. 2. Excluded volume for a node at $\mathbf{z}$ in front of an $L=9$ open-ended chain in a plane. The symbols $\Omega_{1}$ and $\Omega_{2}$ have the same meaning as in Fig. 1. The tube thickness is $D=2.8$ at $R_{0}=1.4$. There are now $9 R_{0}$ circles whose rotations generate the excluded volume in $\mathcal{R}^{3}$, again similar to the construction in Fig. 1 . The cross denotes the position of a possible solution $\mathbf{z}$.

\section{B. Thick polymer models}

The partition function of thick polymers is denoted $Z(D)$ or $Z\left(R_{0}\right)$ with $D=2 R_{0}$ and is obtained upon inserting a $\theta$-function,

$$
\sum_{\text {conf }} 1 \rightarrow \sum_{\text {conf }} \theta\left(R_{g r c}-R_{0}\right)
$$

into the partition function. Alternatively one can use a $\delta$-function constraint

$$
\sum_{\text {conf }} 1 \rightarrow \sum_{\text {conf }} \delta\left(R_{g r c}-R_{0}\right)
$$

For technical reasons we represented $\delta(R)$ by a function which is unity in $[-0.001,+0.001)$, and otherwise zero. We will study ring polymers with partition functions

$$
Z_{\theta}\left(R_{0}\right)=\sum_{\text {rings }} \theta\left(R_{\text {grc }}-R_{0}\right)
$$

where $\theta(z)$ equals 1 for $z \geqslant 0$ and 0 for $z<0$, and

$$
Z_{\delta}\left(R_{0}\right)=\sum_{\text {rings }} \delta\left(R_{g r c}-R_{0}\right)
$$

as well as interacting Lennard-Jones homopolymers

$$
Z_{L J}\left(R_{0}\right)=\sum_{\text {rings }} \delta\left(R_{g r c}-R_{0}\right) e^{-1 / T \Sigma_{i<j+1} V_{L J}\left(r_{i j}\right)}
$$

where $T$ is the temperature. The Lennard-Jones potential has the form

$$
V_{L J}\left(r_{i j}\right)=4\left[\left(\frac{1.6}{2^{1 / 6} r_{i j}}\right)^{12}-\left(\frac{1.6}{2^{1 / 6} r_{i j}}\right)^{6}\right] .
$$

It has a minimum value of -1 at $r_{\min }=1.6$ and thus an openended $L=2$ polymer (three nodes) has a ground state angle of $102.3^{\circ}$ between consecutive bonds. The Lennard-Jones potential is repulsive at short distances, which at low temperatures excludes monomers from the allowed volume along the chain backbone.

The partition functions $Z_{\theta}\left(R_{0}\right)$ and $Z_{\delta}\left(R_{0}\right)$ of Eqs. (2.19) and (2.20) conceptually are somewhat different, in as far as $Z_{\theta}\left(R_{0}\right)$ describes an ensemble with fluctuating $R_{g r c}$ values, while $R_{g r c}$ is fixed within $Z_{\delta}\left(R_{0}\right)$ at $R_{0}$. In addition to that and with the help of $Z_{\delta}\left(R_{0}\right)$, one may also introduce an interesting canonical partition function

$$
Z_{c a n}\left(\eta_{D}\right)=\int d R_{0} Z_{\delta}\left(R_{0}\right) e^{-1 / T \eta_{D} R_{0} L}
$$

as a function of a thermodynamic conjugate variable, $\eta_{D}$, where $\eta_{D}$ is a pressure. The nature of $R_{g r c}$ fluctuations within $Z_{\theta}\left(R_{0}\right)$, however, is quite peculiar: The probability density $p$ to find configurations with probability $P=p \epsilon$ in the vicinity of $R_{0}$, that is in the $R_{g r c}$ value interval $\left[R_{0}, R_{0}+\epsilon\right.$ ) is finite for finite chains and also increases with the chain length. This suggests $Z_{\theta}\left(R_{0}\right)=Z_{\delta}\left(R_{0}\right)$ for infinitely long chains. Currently we cannot prove the equivalence of both partition functions, however, we will show that critical exponent values $\nu_{S A W}$ are independent of the choice $Z_{\theta}\left(R_{0}\right)$ or $Z_{\delta}\left(R_{0}\right)$ at $D=2$. 


\section{Monte Carlo simulation of thick ring polymers}

The Monte Carlo simulation of tubed ring polymers without attractive interactions employs a version of the Madras and Sokal pivot update [22], which is adapted to the geometry of the ring $[23,24]$. Each Monte Carlo step at random chooses a pair $i$ and $j$ of nodes on the chain and then attempts a uniformly distributed large angle rotation, or inversion, of the chain segment between $i$ and $j$ along the direction

$$
\mathbf{e}=\frac{\mathbf{x}_{i}-\mathbf{x}_{j}}{\left|\mathbf{x}_{i}-\mathbf{x}_{j}\right|} .
$$

One can choose $i$ and $j$ to be next to nearest neighbors $j$ $=\bmod (i+2, L)$ on the chain, in which case the resulting local update turns out to be sampling the phase space rather inefficiently. For actual simulations we mostly used a combination of $j=\bmod (i+\Delta L, L)$ with $\Delta L=L / 10$ or $\Delta L=L / 4$ updates and local updates. In a few runs we also use a version where $\Delta L$ was chosen on a random basis from the list $\Delta L$ $=L / 2, L / 3, \ldots, L / 10$. All of these nonlocal updates perform much better than the local one. We did not study algorithmic efficiency in detail, but in general we were satisfied by a huge error reduction when we included nonlocal updates. The error bars of observables were calculated with the help of jackknife binning methods [25]. Finally, our updates employ large-angle rotations and thus the ring knot classes are not conserved.

For ring polymers with attractive interactions we use a combination of the above local pivot updates at $\Delta L=2$ and the nonlocal updates at $\Delta L=L / 10$. Canonical Monte Carlo simulations with the partition function Eq. (2.21) for the Lennard-Jones homopolymer, however, are not quite effective, in particular at low temperatures $T<0.1$. Secondary chain conformations appear to be frozen at these temperatures and the overall chain configuration cannot be thermalized. Clearly a similar problem arises in protein folding simulations where the cure lies in the use of generalized ensemble simulatons, as there are umbrella sampling [26], parallel [27-30] tempering, multicanonical ensemble [31,32], Wang-Landau [33], and multiple Gaussian modified ensemble simulations [34]. All of these Monte Carlo methods attempt to sample a larger phase space volume upon implementing random walks in suitable choosen reaction coordinates. For an open ended Lennard-Jones homopolymer, and in search for ground state configurations of the ring polymer, we have therefore implemented [44] Wang-Landau sampling [33] in the end-to-end distance at the temperature $T=0.1$. In these simulations the end-to-end distance $R_{E T E}$ exhibits random walk behavior, and whenever $R_{E T E}$ is found in the vicinity of unity $1 \leqslant R_{E T E} \leqslant 1.001$ a possibly new, and statistically independent, low-energy configuration for the ring is generated. In a second step then this set of low-energy configurations for rings is iterated to temperature zero at $R_{E T E}=1$ in simulated annealing runs, and several ground state energy estimates are determined. Their minimal value constitutes the best Monte Carlo estimate for the groundstate energy itself.
The basis of all of our Monte Carlo studies is an efficient computation of the global radius of curvature. At first sight this may appear to be a hard task, as naively the calculation requires the evaluation of $O\left(L^{3}\right)$ terms, which exceeds computational resources for long chains. This computational effort can, however, be reduced if one employs geometrical inequalities. Our standard implementation of global radius of curvature calculations (PROJ) uses chain coordinate projections on some arbitrarily choosen coordinate axis and proceeds via the following steps.

(1) First we calculate a guidance value for $R_{\text {grc }}: R_{\text {guide }}$ from the minimum of the set of all local radii of curvature: $R_{r c}\left(\mathbf{x}_{i}, \mathbf{x}_{i+1}, \mathbf{x}_{i+2}\right)$ with $i=1, \ldots, N-2$. This operation takes $O(L)$ operations. Our algorithm will perform the better, the closer $R_{\text {guide }}$ is found above $R_{\text {grc }}$.

(2) We sort one of the chain Cartesian coordinates with respect to their magnitude, e.g., $\mathbf{x}_{i} \cdot \mathbf{e}_{1}$. This operation takes $O(L \ln L)$ calculational steps with algorithms like heap sort. There is also RADIX sort, which performs at $O(k L)$, where $k$ is a key length [35], but whose implementation is more involved.

(3) In the previously sorted list we collect all pairs $i$ and $j$ which satisfy

$$
\left|\left(\mathbf{x}_{i}-\mathbf{x}_{j}\right) \cdot \mathbf{e}_{1}\right|<2 R_{\text {guide }} .
$$

We note that through

$$
\left|\left(\mathbf{x}_{i}-\mathbf{x}_{j}\right) \cdot \mathbf{e}_{1}\right| \leqslant d_{i j} \leqslant 2 R_{r c}\left(\mathbf{x}_{i}, \mathbf{x}_{j}, \mathbf{z}\right)
$$

for any $\mathbf{z}$, only those triangle edges which satisfy Eq. (2.25) can furthermore lower the value $R_{\text {guide }}$ towards $R_{g r c}$. The computational effort pends on the total number of pairs $i$ and $j$ satisfying the condition and in general exceeds $O(L \ln L)$ computational steps.

(4) The previously obtained collection of pairs $i$ and $j$ is filtered to fulfill the remaining orthogonal conditions

$$
\left|\left(\mathbf{x}_{i}-\mathbf{x}_{j}\right) \cdot \mathbf{e}_{2}\right|<2 R_{\text {guide }}
$$

and

$$
\left|\left(\mathbf{x}_{i}-\mathbf{x}_{j}\right) \cdot \mathbf{e}_{3}\right|<2 R_{\text {guide }}
$$

It yields a number of contributing triangle edges, which only scales like $O(L)$.

(5) In a final step, all Euclidean distance squares $d_{i j}^{2}$ of contributing triangle edges are calculated. An additional filter on the length squared $d_{i j}^{2}<4 R_{\text {guide }}^{2}$ reduces the number of contributing triangle edges further. Using Eq. (2.5) we then calculate all squared triangle areas on the set of triangle edges, as well as their corresponding radii of curvature squares. Their minimum can be smaller than $R_{\text {guide }}^{2}$ and the final $R_{g r c}^{2}$, respectively, $R_{g r c}$ value is determined. Discarding local triangles attached to local radii of curvature, we measure the number $\bar{N}_{N L}$ of nonlocal triangles in this step in the mean. Our numerical finding suggests a linear dependence of $\bar{N}_{N L}$ on the chain length for ring polymers without attractive interactions, as well as for Lennard-Jones homopolymer rings with attraction. For purposes of illustration we collect in Table I measurements of $\bar{N}_{N L}$ for the case of LennardJones homopolymers at $R_{g r c}=1.4$ and $T=0.1$. The third row 
TABLE I. Mean number $\bar{N}_{N L}$ of nonlocal triangle calculations, as described in the text. We present data for the Lennard-Jones homopolymer at $R_{g r c}=1.4(D=2.8)$ and $T=0.1$ as a function of the length $L$ of the ring.

\begin{tabular}{lcc}
\hline \hline$L$ & $\bar{N}_{N L}$ & $\bar{N}_{N L} / L$ \\
\hline 20 & 42.5 & 2.12 \\
30 & 56.5 & 1.88 \\
40 & 70.1 & 1.75 \\
50 & 89.7 & 1.79 \\
60 & 109.8 & 1.83 \\
70 & 127.2 & 1.83 \\
80 & 136.8 & 1.71 \\
90 & 154.5 & 1.72 \\
100 & 170.8 & 1.71 \\
\hline \hline
\end{tabular}

of the table contains $\bar{N}_{N L}$ in units of $L$, which for the largest ring length values appears to stabilize at values $\bar{N}_{N L} / L$ $\approx 1.7$. It is also instructive to present some numbers for global radius calculations of the Lennard-Jones homopolymer ring at $L=100$. Typical configurational radius of curvature values within $Z_{\theta}$ turn out to be numbers like $1.4029,1.4011$, or 1.4002 . In about $50 \%$ of the cases $R_{\text {guide }}$ is actually larger than $R_{\text {grc }}$, but not far away, a typical value is $R_{\text {guide }}=1.4044$. After sorting and upon imposing Eq. (2.25), we find a number of edges which is 2277 in the mean. Conditions, Eqs. (2.27) and (2.28), reduce that number to about 756 in the mean, while $d_{i j}^{2}<4 R_{\text {guide }}^{2}$ yields a number of 319 contributing triangle edges. Apart from $L+1$ local radii of curvature calculations in the guidance value $R_{\text {guide }}$ determination step, we then have to evaluate about 171 nonlocal radii of curvature and their associated triangles in the mean, as can be found in Table I.

Our algorithm uses the apparent fact that once the guidance value $R_{\text {guide }}$ is known, only triangle edges with

$$
\left|\mathbf{x}_{i}-\mathbf{x}_{j}\right|<2 R_{\text {guide }}
$$

can contribute. A similar problem arises in atomistic molecular dynamics simulations with finite interaction range and the preferred solution method there is the cell list method [36]. The cell list method (CELL) maps the chain coordinates to cubic cells of linear extent $2 R_{\text {guide }}$ and uses cell neighborhood relations to solve Eq. (2.29), while PROJ employs a similar idea in one of the projections of the coordinates. In Fig. 3 we compare PROJ and CELL computer times $\tau_{1 M E G A}$ in CPU seconds for the actual calculation of $10^{6}$ global radii of curvature. These benchmarks were performed on a modern 2.4 Ghz Opteron workstation. Figure 3(a) displays data from simulations at $R_{g r c}=1.0$ for long and rather dilute rings without Lennard-Jones potential as a function of $L$, while Fig. 3(b) contains similar data for somewhat shorter and denser Lennard-Jones homopolymers at $R_{g r c}=1.4$ and $T=0.1$. For both cases and for the given parameter range CELL (the triangles in the figure) and PROJ (the circles in the figure) computer times are quite close to each other. The solid curves
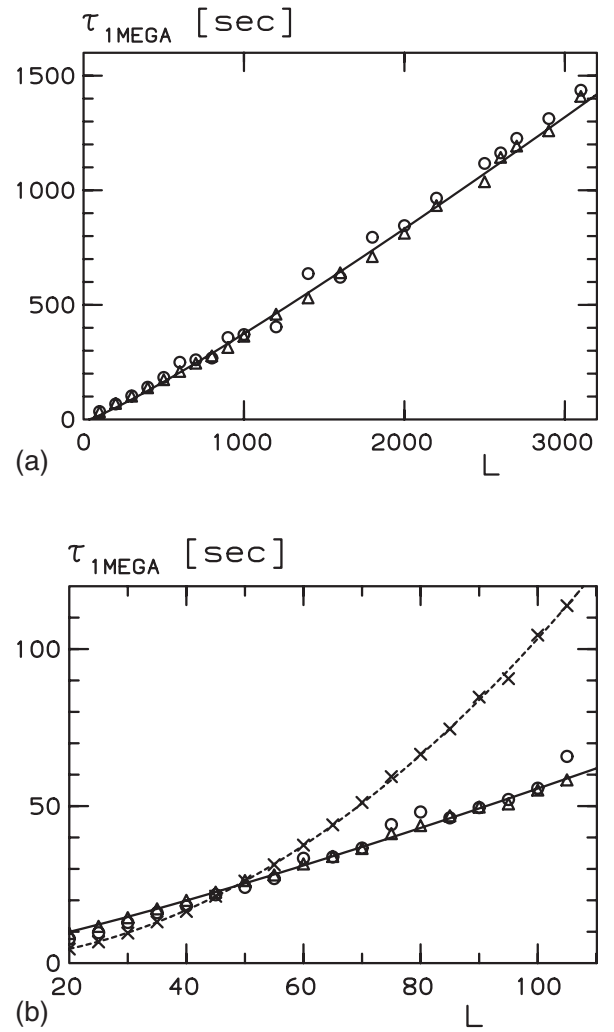

FIG. 3. Computer times $\tau_{1 M E G A}$ in seconds for the calculation of $10^{6}$ global radii of curvature as a function of $L$ at $D=1$ in (a) and $D=2.8$ in (b). CELL list algorithm data are marked by triangles, while PROJ algorithm data are given by circles. The curves through triangles and circles describe a $L \ln L$ singularity. Panel (b) also contains computer times for the evaluation of $10^{6}$ Lennard-Jones energies (crosses), which as indicated by the dashed curve increase $\propto L^{2}$.

within the figures correspond to minima of the form $\sum_{i}\left(\tau_{1 M E G A, i}-\tau_{1 M E G A}\right)^{2}$ with $\tau_{1 M E G A, i}$ denoting the measured CELL data. The cell list method yields under optimal conditions the dependency

$$
\tau_{1 M E G A}=A L \ln L+\text { const }
$$

of the computer time effort $\tau_{1 M E G A}$ on the chain length $L$ and the data are consistent with such a behavior. In the case of the diluted long rings we obtain $A(\mathrm{CELL})=0.055$ in units of CPU seconds per chain length, and $A(\mathrm{CELL})=0.114$ for the denser Lennard-Jones homopolymers. The PROJ algorithm performs at the same level of efficiency for the considered chains. We obtain $A(\mathrm{PROJ})=0.057$ and $A(\mathrm{PROJ})=0.127$, respectively. Figure 3(b) also contains a measurement of computer times for the evaluation of $10^{6}$ Lennard-Jones potential energies, which as expected diverge proportional to $L^{2}$ and therefore exceed global radius of curvature calculations for $L$ values larger than about 50 . In total the computational efficiency of global radius of curvature calculations is established, and from a technical point of view such calculations can easily be included into the modeling of less simplistic polymer models like heteropolymers, or even into models of 

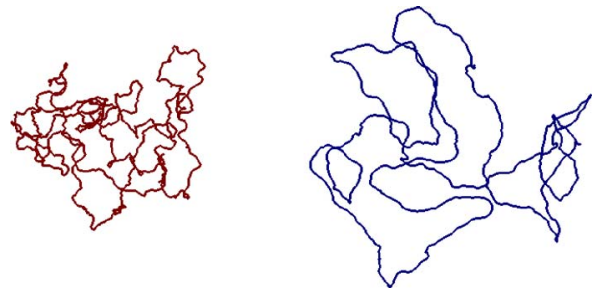

FIG. 4. (Color online) Two ring configurations of tubes for $L$ $=1000$ at $R_{g r c}=1.0$ (on the left) and $R_{g r c}=2.0$ (on the right). The authors have inspected these configurations by eye. The finding is that these rings are in the trivial knot class, even though the nonlocal updates are capable of achieving nontrivial knots and actually sum over all knot classes.

proteins. The computer codes of PROJ and CELL may be obtained from the authors.

\section{RESULTS OF SIMULATIONS}

We simulated thick tubes using $Z_{\theta}\left(R_{g r c}\right)$ at the values $R_{g r c}=0.25$ and 0.48 within the "unphysical" region and at $R_{g r c}=1.0$ and 2.0 on rings with $L$ values ranging between $L=50$ and 4000. At $R_{g r c}=1.0$ an additional study with $Z_{\delta}\left(R_{g r c}\right)$ was performed. All of our simulations are done at the length scale $a=1$ in the discrete tube model. In Fig. 4 we show two $L=1000$ ring configurations at the values $R_{g r c}$ $=1.0$ and 2.0. It can be seen that increasing tube thickness drives the geometrical scales of ring configurations to larger values.

\section{A. Scaling of thick ring polymers}

In the Monte Carlo simulation we measure the mean radius of gyration $R_{g}$ squared, which is defined as

$$
R_{g}^{2}=\frac{1}{L}\left\langle\sum_{i}\left(\mathbf{x}_{i}-\mathbf{x}_{c m s}\right)^{2}\right\rangle
$$

with the center of mass

$$
\mathbf{x}_{c m s}=\frac{1}{L} \sum_{i} \mathbf{x}_{i} .
$$

Its root $\sqrt{R_{g}^{2}}$ is $R_{g}$. We display in Fig. 5 all of our $R_{g}$ simulation data within the $Z_{\theta}$ ensmble in units of $R_{g r c}$. The curves in the plot correspond to the finite length scaling forms $R_{g}$ $\propto L^{\nu_{S A W}}$ with a fixed value $\nu_{S A W}=0.5874$. None of the data sets at either value of $R_{g r c}$ is consistent with a Gaussian behavior at $\nu=0.5$. The theoretically predicted asymptotic scaling form is

$$
R_{g}=A L^{\nu_{S A W}}\left(1+\frac{B}{L^{\Delta}}\right)
$$

and therefore the effective exponent quantity

$$
\nu_{S A W, e f f}=\frac{1}{\ln (2)} \ln \left(\frac{R_{g}(L)}{R_{g}(L / 2)}\right)
$$

approaches $\nu_{S A W}$ in the thermodynamic limit. The scaling corrections are

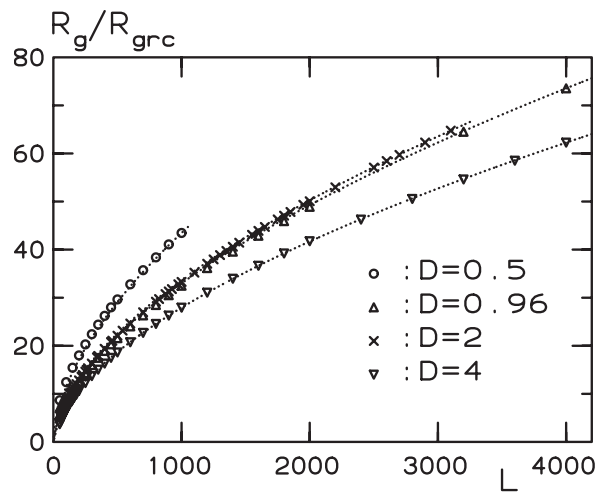

FIG. 5. All $R_{g}:=\sqrt{R_{g}^{2}}$ data of Eq. (3.1) in units of $R_{g r c}$ at $R_{g r c}$ $=0.25,0.48$, and 1.0 and $R_{g r c}=2.0$ with $D=2 R_{g r c}$ as a function of $L$. The scaling curves use $\nu_{S A W}=0.5874$ and are adjusted to match the data points at largest $L$. Around $D \approx 1$ to 2 the polymer swelling is const $\times D$

$$
\nu_{S A W, e f f}(L)=\nu_{S A W}+\frac{C}{L^{\Delta}}
$$

with a value of $\Delta \approx 0.5$. In Fig. 6 , we show $\nu_{S A W, e f f}$ data from $Z_{\theta}$ simulations as a function of $1 / \sqrt{L}$ for three global radius of curvature values $R_{g r c}=0.25,0.48$, and 2.0. All data are inconsistent with a Gaussian value $\nu_{S A W}=0.5$, but can approach the value $\nu_{S A W}=0.5874$, the horizontal line in the figure. Scaling corrections are, however, particularly large at $R_{g r c}=0.25$, or are even nonmonotonic for the parameter values $R_{g r c}=0.48$ and 2.0. The $R_{g r c}=0.48$ run exhibits rather small scaling corrections, yielding $\nu_{S A W, \text { eff }} \approx 0.588(3)$ from the matching of the $L=4000$ and 2000 chains. A final decision for these global radii of curvature values requires even longer chains, which are not currently accessible with our methods. The simulation on the $L=4000$ chain at $R_{g r c}$ $=0.48$ took about 1 week on a modern workstation and its error bar is still too large. The situation turns out to be much

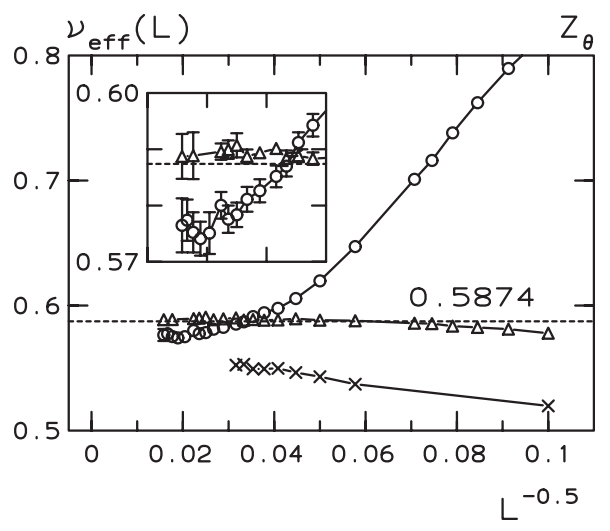

FIG. 6. Effective exponent data of Eq. (3.4) at $R_{g r c}=0.25$ (crosses), $R_{g r c}=0.48$ (triangles), and $R_{g r c}=2.0$ (circles) with $D$ $=2 R_{\text {grc }}$ as a function of $1 / \sqrt{L}$. The dashed horizontal lines are at $\nu_{S A W}=0.5874$. These data points either show large scaling deviations or even indicate a nonmonotonic trend in the final approach of $\nu_{\text {eff }}(L)$ towards $L \rightarrow \infty$. The inlay magnifies the large $L$ behavior of $R_{g r c}=0.48$ and $R_{g r c}=2.0$ data. 
TABLE II. Observable values $R_{g}=\sqrt{R_{g}^{2}}$ with $R_{g}^{2}$ as of Eq. (3.1) and number of Monte Carlo steps in units of $10^{6}$, for $Z_{\theta^{-}}$-simulation data at $R_{g r c}=1.0$ as a function of $L$. The last row denotes an absolute $\delta R_{g}$ statistical error, at the statistics of $10^{9}$ Monte Carlo steps. Most, but not all, simulations were performed with the pivot nodes $j$ $=\bmod (i+L / 10, L)$.

\begin{tabular}{lccc}
\hline \hline$L$ & $R_{g}$ & MC - steps $\times 10^{-6}$ & $\delta R_{g}$ \\
\hline 800 & $29.1766(0147)$ & 779 & 0.0129 \\
825 & $29.7249(0022)$ & 2307 & 0.0034 \\
875 & $30.7606(0054)$ & 2314 & 0.0082 \\
900 & $31.2987(0229)$ & 619 & 0.0180 \\
925 & $31.7989(0039)$ & 2235 & 0.0058 \\
975 & $32.7923(0040)$ & 3313 & 0.0073 \\
1000 & $33.2932(0127)$ & 1494 & 0.0155 \\
1100 & $35.2142(0034)$ & 2290 & 0.0051 \\
1200 & $37.0666(0059)$ & 2377 & 0.0091 \\
1250 & $37.9681(0053)$ & 2182 & 0.0079 \\
1300 & $38.8641(0079)$ & 2069 & 0.0114 \\
1350 & $39.7346(0037)$ & 2079 & 0.0053 \\
1400 & $40.5965(0072)$ & 1973 & 0.0102 \\
1450 & $41.4432(0063)$ & 1994 & 0.0089 \\
1550 & $43.0783(0032)$ & 1923 & 0.0045 \\
1600 & $43.9095(0085)$ & 1693 & 0.0110 \\
1650 & $44.7133(0087)$ & 1813 & 0.0117 \\
1750 & $46.2819(0083)$ & 1778 & 0.0111 \\
1800 & $47.0650(0091)$ & 2331 & 0.0139 \\
1850 & $47.8332(0084)$ & 1677 & 0.0109 \\
1950 & $49.3343(0117)$ & 1625 & 0.0149 \\
2000 & $50.0607(0104)$ & 2164 & 0.0153 \\
2200 & $52.9607(0100)$ & 1443 & 0.0120 \\
2500 & $57.0701(0157)$ & 1288 & 0.0178 \\
2600 & $58.4337(0142)$ & 1233 & 0.0158 \\
2700 & $59.7140(0137)$ & 1194 & 0.0150 \\
2900 & $62.2928(0216)$ & 1084 & 0.0224 \\
3100 & $64.7584(0163)$ & 1015 & 0.0164 \\
\hline \hline
\end{tabular}

more comfortable at the global radius of curvature value $R_{g r c}=1.0$. For this curvature value, independent simulations were performed within $Z_{\theta}$, see Table II, and $Z_{\delta}$. The corresponding $\nu_{S A W, \text { eff }}$ values again are plotted as a function of $1 / \sqrt{L}$ in Figs. 7 and 8 (higher resolution) for the $Z_{\theta}$ case and in Fig. 9 for the $Z_{\delta}$ case. Chain length values range from $L$ $=50$ to 3100 . Including leading, and subleading, scaling and scaling corrections as in Eq. (3.5) a three-parameter fit to a number of 28 finite length effective exponent data at all $L$-values yields

$$
\nu_{S A W, e f f}(L)=0.5866(15)+\left(\frac{6.7(7)}{L^{1.06(5)}}\right)
$$

at $\chi_{\text {d.o.f. }}^{2}=1.05$ for $Z_{\delta}$ data, while a fit to a number of 39 data points gives

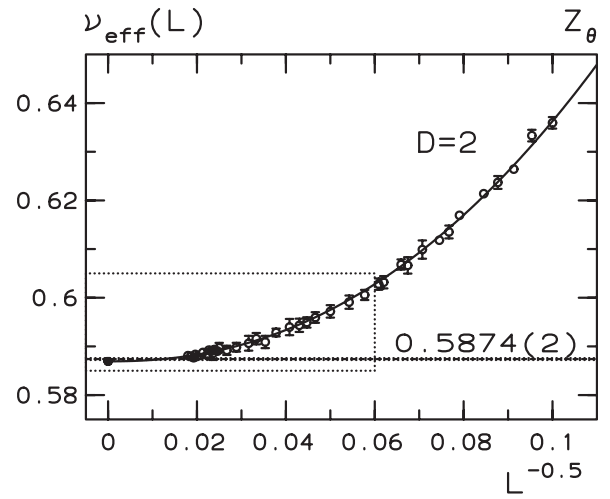

FIG. 7. Effective exponent data of Eq. (3.4) at $R_{g r c}=1.0$ or $D$ $=2$ from $Z_{\theta}$ simulations as a function of $1 / \sqrt{L}$. The horizontal line is at $\nu_{S A W}=0.5874$. A three-parameter fit of corresponding $\nu_{\text {eff }}$ data to the form Eq. (3.5) yields the parameters in Eq. (3.7). The curve corresponds to the fit and the data point at zero corresponds to the extrapolated $\nu_{S A W}$ value. The plotted rectangle marks the outer limits of a higher resolution figure: Fig. 8.

$$
\nu_{S A W, e f f}(L)=0.5869(05)+\left(\frac{8.2(9)}{L^{1.11(3)}}\right)
$$

at $\chi_{\text {d.o.f. }}^{2}=0.72$ for $Z_{\theta}$. Hereby $\chi_{\text {d.o.f. }}^{2}$ denotes the $\chi^{2}$ value per degree of freedom (d.o.f.) of corresponding fits. The $Z_{\theta}$ simulation has much better statistics and thus we quote the final result

$$
\nu_{S A W}=0.5869(5),
$$

which is entirely consistent with the $Z_{\delta}$ result, and the best current on-lattice determinations $\nu_{S A W}=0.5877(6) \quad$ [18], $\nu_{S A W}=0.5874(2)[19], \nu_{S A W}=0.58765(20) \quad[20]$, and $\nu_{S A W}$ $=0.58758(7)$ [21]. For a review of further numerical and analytical $\nu_{S A W}$ calculations see [37]. A few remarks are in order.

(1) We have argued that statistical theories built around three-point functions must be viewed as effective theories. The three-point property of global radii prohibits a factorization of the tubes partition function into two-body potentials [38]. It then appears as if one condition for the canonical perturbative treatment of self-avoidance [39] is not fulfilled. Nevertheless, the measurements at $D=2$ and 0.96 yield con-

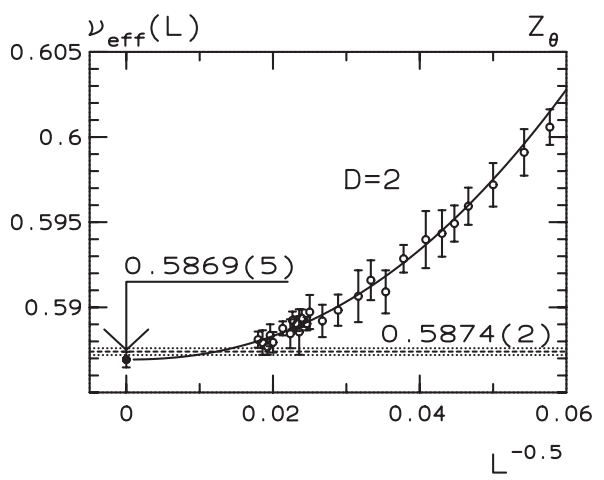

FIG. 8. Finer-scale representation of effective exponent data at $R_{g r c}=1.0$ or $D=2$ from $Z_{\theta}$ simulations as a function of $1 / \sqrt{L}$. 


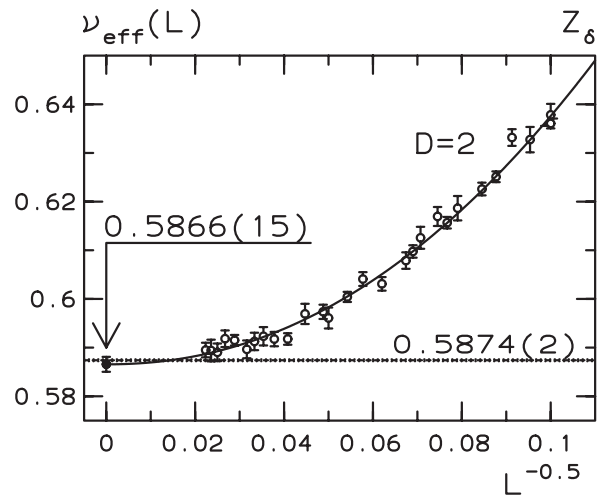

FIG. 9. Effective exponent data of Eq. (3.4) at $R_{g r c}=1.0$ or $D$ $=2$ from $Z_{\delta}$ simulations as a function of $1 / \sqrt{L}$. The horizontal line is at $\nu_{S A W}=0.5874$. A three-parameter fit of corresponding $\nu_{\text {eff }}$ data to the form Eq. (3.5) yields the parameters in Eq. (3.6). The curve corresponds to the fit and the data point at zero corresponds to the extrapolated $\nu_{S A W}$ value.

sistent results for the tubes Flory exponent $\nu_{S A W}$ with field theory $\nu \approx 0.5874$. Within the statistical ensemble, the threepoint functions of the global radius of curvature are effectively reduced to two-point functions with the universality class of a physical, i.e., self-avoiding ring [12-14]. We also find large deviations from a Gaussian behavior even deep in the "unphysical" region of the ring at $D=0.5$ and a similar statement is likely to apply there.

(2) Corrections to scaling of the self-avoiding ring are expected to be of a similar form as the corrections to scaling of an open-ended self-avoiding-walk $[13,14]$. The fitted value $\Delta_{\text {ring/tube }} \approx 1.1$ for the rings leading scaling corrections at $D=2.0$ is, however, not consistent with the universal field theory prediction $\Delta \approx 0.5$. On the other hand, and for the case of open-ended self-avoiding-walks, several researchers have reported on the existence of "magic points" [11,40-42], where leading scaling corrections would vanish, and where subleading scaling correction terms dominate. (Baumgärtner in [40] puts the "magic point" at $h / a \approx 0.55$ in an off-lattice hard-sphere polymer model, where $a$ denotes the segment length and $h$ the sphere diameter. This corresponds to a relatively thin tube of ours and is away from the $D=2$ region.) Our numerical findings at $D=2$ are in support of the existence of such a "magic point" for the self-avoiding ring with a location at thickness values around $D \approx 2.0$. The precise locations of "magic points," as well as their properties, to our knowledge have not yet been predicted by theory for the ring-polymer case. They can only be obtained through additional numerical simulations.

\section{B. Ground-state configurations of interacting tubes}

We also explore the properties of low-temperature and near-ground-state conformations of interacting flexible thick rings at a value $R_{g r c}=1.4$. At low temperatures-we choose $T=0.1$ in our simulations-the attractive Lennard-Jones potential favors a collapsed polymer ring, while the tube thickness operates in the opposite direction. The resulting ring polymers are less compact than zero thickness polymers, i.e.,

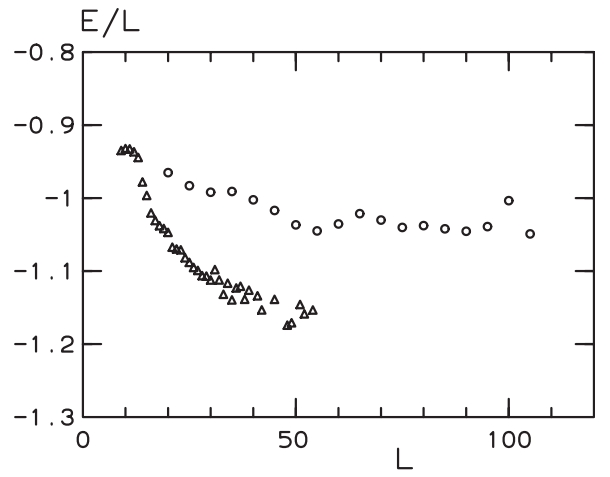

FIG. 10. Low-temperature energy densities from $T=0.1$ simulations (circles) and near-ground-state energy densities at $T \approx 0$ (triangles) for the $R_{g r c}=1.4$ Lennard-Jones ring homopolymer as a function of $L$. The data points at $T=0.1$ stem from Monte Carlo simulations of the partition function Eq. (2.21), where in long runs the minimal potential energy value was recorded. Near-ground-state energy densities at $T \approx 0$ were obtained from an extensive Monte Carlo search in phase space as explained in Sec. II C. We cannot easily quote statistical error bars, as in both cases the observable arrives as the minimum value of a Monte Carlo time series. The scattering of $T \approx 0$ energy density values at large $L$ reflects the inability of the simulation to obtain the exact ground state.

marginally compact, but considerably more compacted than tubes without attractive interactions. In the simulations we measure the Lennard-Jones potential energy $E_{L J}$ $=\sum_{i<j+1} V_{L J}\left(r_{i j}\right)$ and from long Monte Carlo runs we determine a single minimal $E_{L J, \min }$ value, as well as the corresponding chain configuration. The energy densities of these low-temperature configurations correspond to circles in Fig. 10 for chain length values $L=20, \ldots, 105$.

A determination of the true homopolymer ground state is an extremely ambitious project, even for chains of moderate length, e.g., chains of length $L=32$, if the chain is described by a continuous set of coordinates. In a related project [44], we developed efficient Monte Carlo algorithms which search the phase space for near-ground-state configurations and near-ground-state energies in a much better way. We note that from a technical point of view, any Monte Carlo search on a continuous set of coordinates is bound to fail in finding the exact ground state, while near-ground-state properties can be retrieved. Some results for near-ground-state energy densities $E_{L J, 0} / L$ of the Lennard-Jones homopolymer ring at $R_{g r c}=1.4$ are displayed in Fig. 10 (the triangles in the figure) for chains of length $L=9-54$. We note that within the considered length interval none of the energy densities for large $L$ has settled to a trustworthy constant value. The infinite and finite length homopolymer ground state and its energy is extremely hard to calculate, even with somewhat improved algorithms.

Here in Figs. 11 and 12 we show two low-temperature $(T=0.1)$ configurations for interacting Lennard-Jones ring homopolymers at lengths $L=95$ and 105, respectively. Each figure contains a 3D plot of the full ring at the top left corner (position 1) and at positions 2, 3, 4, and 5 (counted clockwise from the top left corner) a plot of some local substructures within the same ring. 


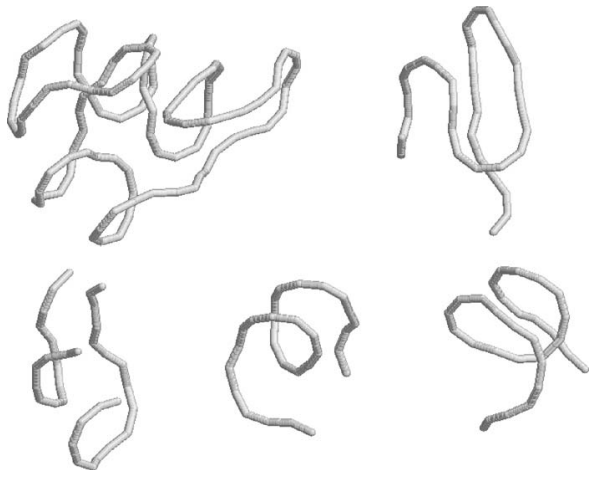

FIG. 11. Low-temperature conformations of a single LennardJones ring homopolymer from $T=0.1$ simulations at $R_{g r c}=1.4$ for $L=95$. The top left corner (at position 1) displays the complete ring, while as counted clockwise from the top left corner, positions 2, 3, 4 , and 5 display substructures of the same ring.

Within inhomogeneous chain configurations as displayed in Figs. 11 and 12, there will be chain sections, or multiple sections of the chain, which in energy are more strongly bound than others at less tight binding. Using binding energy values $E_{L J}(i, j)$ in-between monomers at chain positions $i$ and $j$, we construct a two dimensional interaction map in $i$ and $j$, which only has nonvanishing entries, if $E_{L J \text {,min }}$ $\leqslant E_{L J}(i, j)<E_{c u t} . E_{L J, m i n}$ denotes the minimum of all $E_{L J}(i, j)$. A sensible choice of the parameter value $E_{c u t}$, in union with a nearest neighbor connectivity definition in $i$ - $j$ space, then defines several disconnected cluster components, which can be identified. These correspond to the local substructures under discussion. On a descriptive level, we only find a few short chain conformational building blocks, which are present at low temperature:

(1) The $L=95$ ring in Fig. 11 contains two helices of opposite helicity, positions 3 and 4 in the figure. The helical cylinder radius is $R_{\text {helix }} \approx R_{\text {grc }}$. Helices are short and exhibit 1 to 2 windings.

(2) Both the $L=95$ ring and the $L=105$ ring contain several U-turns with turn radii $R_{U \text {-turn }} \approx R_{\text {grc }}$, positions 2 and 5 in Fig. 11 as well as positions 2 and 3 in Fig. 12. There are occurrences where the chain sequence consists of several

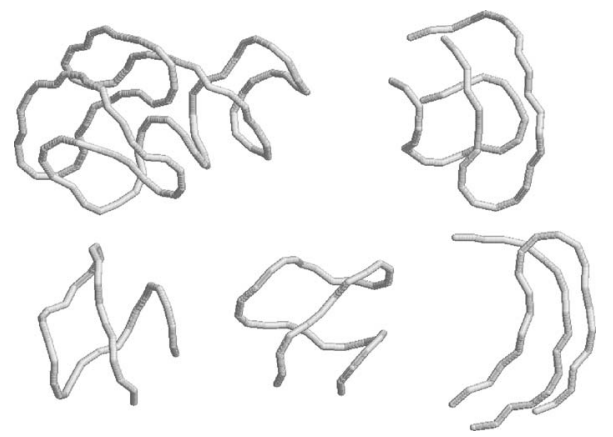

FIG. 12. Low-temperature conformations of a single LennardJones ring homopolymer from $T=0.1$ simulations at $R_{g r c}=1.4$ for $L=105$. The top left corner (at position 1) displays the complete ring, while as counted clockwise from the top left corner, positions 2-5 display substructures of the same ring. consecutive U-turns, which in 3D form pairs standing faceto-face at distance $D=2 R_{g r c}$, see position 2 in Fig. 11 . The periodic repetition of U-turns with face-to-face binding resembles a crystalline structure.

(3) There are several occurrences of U-turns, positions 2 and 3 in Fig. 12, that are connected to extended pairs of chain segments, which have small local curvature, i.e., are rather straight and which are parallel at distance $D=2 R_{g r c}$. These structures appear to screen any curved inner ring structure on the surface of the fold.

The advance of a likewise finite set of short chain generic conformational building blocks in low-temperature and nearground-state configurations of thick polymers was conjectured to be a universal feature within a whole class of attractive homopolymer interactions. This appears to be a plausible conjecture, as in this view ground states of short homopolymers are near the dense packing limit of flexible tubes, and thus some geometrically induced universality can be present. In addition, any extended long chain and nearground-state configuration is a mixture-or mixed phase-of short-chain conformational building blocks, as shown in Figs. 11 and 12. This scenario has been strongly advocated in [3-5] and is also supported by the simulation of small alanine-based artificial peptides [43]. We are currently classifying short-chain conformational building blocks for various forms of thick tube chain attractions. Some of our conformations appear to differ from those presented [38], which may be caused by the use of different potential functions and Monte Carlo sampling schemes.

\section{CONCLUSION}

Thick ring polymers with nonattractive interactions can be efficiently simulated in Monte Carlo simulations of offlattice chains up to chain length $L=4000$ if nonlocal chain updates and improved algorithms for the calculation of the global radius of curvature are employed. In the fieldtheoretic limit of long chains at thickness $D=2.0$ we determine a critical "correlation length" scaling exponent $\nu_{S A W}$ $=0.5869(5)$, which puts the thick ring polymer without attractive interactions into the 3D universality class of the selfavoiding walk. The numerical quality of our data is competitive with other numerical simulations. Rings of tubes at $D$ $=2.0$ appear to have scaling corrections which decay faster than in the case of other self-avoiding chains, possibly induced by the proximity of a "magic point." Our $\nu_{S A W}$ study apparently succeeds for smaller chain length values $[L$ $=3100$ in our case, instead of $L=80000$ in [9] at $\nu_{S A W}$ $=0.5877(6)]$. Naturally, and as in all Monte Carlo studies at a finite number of degrees of freedom, the stability of the findings ought to be checked under increasing numbers. We also studied some other values of the thickness $D=0.5,0.96$, and $D=4$, but nonmonotonic or large scaling corrections prevent a stringent test of universality, even though our data are consistent with universal behavior. Within the partition function we also find that slightly different implementation thickness constraints do not affect the final results on $\nu_{S A W}$.

It is conjectured that near-ground-state chain configurations of short thick tubes with attractive homopolymer 
interactions would only fold into a finite set of conformational building blocks [3-5]. For a particular form of the Lennard-Jones potential, and in an exploratory study, we have presented a possibly incomplete list of some of these conformational building blocks, like short helices and U-turns. The degree of universality in these structures, as well as their detailed geometrical properties are currently under investigation [44].

\section{ACKNOWLEDGMENTS}

T.N. thanks Professor P. Grassberger for various discussions on the subject and appreciates insights into the cell list method conveyed by Dr. S. Mohanty. The calculations were done on computers of the John von Neumann Institute for Computing, Forschungszentrum Jülich, Jülich, Germany. This work was supported, in part, by a research grant (CHE0313618) from the National Science Foundation (USA).
[1] U. H. E. Hansmann, Comput. Sci. Eng. 5, 64 (2003).

[2] P. E. Leopold, M. Montal, and J. N. Onuchic, Proc. Natl. Acad. Sci. U.S.A. 89, 8721 (1992).

[3] J. R. Banavar and A. Maritan, Rev. Mod. Phys. 75, 23 (2003).

[4] J. R. Banavar, A. Flammini, D. Marenduzzo, A. Maritan, and A. Trovato, J. Phys.: Condens. Matter 15, 1787 (2003).

[5] J. R. Banavar, T. X. Hoang, A. Maritan, F. Seno, and A. Trovato, Phys. Rev. E 70, 041905 (2004)

[6] U. H. E. Hansmann, J. Chem. Phys. 120, 417 (2004).

[7] C.-Y. Lin, C.-K. Hu, and U. H. E. Hansmann, Proteins: Struct., Funct., Genet. 52, 436 (2003).

[8] O. Gonzalez and H. Maddocks, Proc. Natl. Acad. Sci. U.S.A. 96, 4769 (1999).

[9] T. Ashton and J. Cantarella, e-print arXiv:math-ph/0409416.

[10] R. B. Kusner and J. M. Sullivan, in Topology and Geometry in Polymer Science (Minneapolis, 1996), IMA Volumes in Mathematics and Its Applications, Vol. 103, edited by S. G. Whittington, W. D. Sumners, and T. Lodge (Springer, New York, 1998).

[11] O. Krötenheerdt and S. Veit, Beitr. Algebra Geom. 5, 61 (1976)

[12] A. Baumgärtner, J. Chem. Phys. 76, 4275 (1982); P. D. Gujrati, Phys. Rev. B 27, 4507 (1983).

[13] J. J. Prentis, J. Phys. A 17, 1723 (1984).

[14] P. Calabrese, A. Pelissetto, and E. Vicari, J. Chem. Phys. 116, 8191 (2002).

[15] K. Koniaris and M. Muthukumar, Phys. Rev. Lett. 66, 2211 (1991).

[16] M. Brill, P. M. Diesinger, and D. W. Heermann, e-print arXiv:cond-mat/0507020.

[17] P. Virnau, L. A. Mirny, and M. Kardar, PLOS Comput. Biol. 2, 1074 (2006).

[18] B. Li, N. Madras, and A. D. Sokal, J. Stat. Phys. 80, 661 (1995).

[19] T. Prellberg, J. Phys. A 34, L599 (2001).

[20] H.-P. Hsu, W. Nadler, and P. Grassberger, Macromolecules 37, 4658 (2004).

[21] P. Belohorec and B. Nickel (unpublished).

[22] N. Madras and A. Sokal, J. Stat. Phys. 50, 109 (1988).

[23] N. Madras, A. Orlitsky, and L. A. Shepp, J. Stat. Phys. 58, 159
(1990); S. Caracciolo, A. Pelissetto, and A. D. Sokal, ibid. 60, 1 (1990); E. J. Janse van Rensburg, S. G. Whittington, and N. Madras, J. Phys. A 23, 1589 (1990).

[24] J. M. Deutsch, Phys. Rev. E 59, R2539 (1999).

[25] B. A. Berg, Comput. Phys. Commun. 69, 7 (1992).

[26] G. M. Torrie and J. P. Valleau, J. Comput. Phys. 23, 187 (1977).

[27] C. G. Geyer, in Computing Science and Statistics, Proceedings of the 23rd Symposion on the Interface, edited by E. M. Keramidas (Interface Foundation, Fairfax, VA, 1991), pp. 156-163.

[28] A. P. Lyubartsev, A. A. Martsinovski, S. V. Shevkanov, and P. N. Vorontsov-Velyaminov, J. Chem. Phys. 96, 1776 (1992).

[29] E. Marinari and G. Parisi, Europhys. Lett. 19, 451 (1992).

[30] K. Hukusima and K. Nemoto, J. Phys. Soc. Jpn. 65, 1604 (1996).

[31] B. A. Berg and T. Neuhaus, Phys. Lett. B 267, 249 (1991).

[32] B. A. Berg and T. Neuhaus, Phys. Rev. Lett. 68, 9 (1992).

[33] F. Wang and D. P Landau, Phys. Rev. E 64, 056101 (2001).

[34] T. Neuhaus and J. S. Hager, Phys. Rev. E 74, 036702 (2006).

[35] D. E. Knuth, Sorting and Searching, Volume 3 of The Art of Computer Programming (Addison-Wesley, Reading, MA, 1973).

[36] R. W. Hockney and J. W. Eastwood, in Computer Simulations Using Particles (McGraw-Hill, New York, 1981).

[37] A. Pelissetto and E. Vicari, Phys. Rep. 368, 549 (2002).

[38] D. Marenduzzo, A. Flammini, A. Trovato, J. R. Banavar, and A. Maritan, J. Polym. Sci., Part B: Polym. Phys. 43, 650 (2005).

[39] H. Kleinert, in Path Integrals in Quantum Mechanics, Statistics, Polymer Physics, and Financial Markets 4th ed. (World Scientific, Singapore, 2004).

[40] A. Baumgärtner, J. Chem. Phys. 76, 4275 (1982).

[41] B. Krüger and L. Schäfer, J. Phys. I 4, 757 (1994); Macromolecules 29, 4737 (1996).

[42] L. Lue and S. B. Kiselev, J. Chem. Phys. 111, 5580 (1999).

[43] N. A. Alves and U. H. E. Hansmann, J. Phys. Chem. 117, 2337 (2002).

[44] T. Neuhaus, Ulrich H. E. Hansmann, and O. Zimmermann (in preparation). 